PACS numbers: 68.35.Bs, 68.35.Dv, 68.35.Gy, 68.35.Ja, 68.35.Rh, 75.70.Rf

\title{
Surface Reconstruction: Noble Metals
}

\author{
M. A. Vasyl'yev, A. B. Bondarchuk, and V. A. Tinkov \\ G. V. Kurdyumov Institute for Metal Physics, N.A.S. of the Ukraine, \\ 36 Academician Vernadsky Blvd., UA-03680 Kyyiv-142, Ukraine
}

The paper reviews the most important achievements in the study of the surfacereconstruction phase transition on the low-index single crystals of Pt, Ir, and $\mathrm{Au}$. A number of methods very sensitive to the surface layers are used to study the stable and metastable structures of the (100), (110), and (111) faces. Detailed consideration of the kinetics, critical parameters, activation energy, and geometrical models for 2D-surface phase transitions are presented. Main theoretical results based on the Ising 2D-model, calculations of the free energy with an embedded-atom method, and equilibrium Monte Carlo simulations are also given.

В обзоре рассмотрены последние достижения в исследовании реконструкционных фразовых превращений в поверхностных слоях монокристаллов $\mathrm{Pt}$, Ir и Au. Приведены результаты, полученные с помощью различных поверхностно-чувствительных экспериментальных методов. Наиболее подробно рассмотрены вопросы, относящиеся к кинетике, энергии активации, геометрическим моделям, критическим параметрам 2D-сразовых превращений. Представлены также результаты теоретических исследований, основанных на 2D-модели Изинга, методе внедренного атома и моделировании методом Монте-Карло.

В обзорі розглянуто останні досягнення в дослідженні реконструкційніх фразових перетворень в поверхневих шарах монокристалів $\mathrm{Pt}$, Ir та $\mathrm{Au}$. Haведено результати, які отримані за допомогою різних повехнево-чутливих експериментальних методів. Найбільш детально розглянуто питання, що відносяться до кінетики, енергії активації, геометричних моделей, критичних параметрів 2D-фазових перетворень. Представлено також результати теоретичних досліджень, що базовані на 2D-моделі Ізінга, методі зануреного атому та моделюванні за методом Монте-Карло.

Key words: surface phase transition, surface reconstruction, low-energy electron diffraction, Ising model, 2D-symmetry.

(Received October 27, 2000) 


\section{INTRODUCTION}

The determination of the atomic structure of metal surfaces, and adsorbed phase continues to be the central problem in surface science. Clearly, an understanding of the complex processes involved, for example, in chemisorption and catalysis requires a detailed knowledge of the positions of substrate and adsorbate atoms. It is well known that the atomic structure of the clean single-crystal metal surfaces may be different from that obtained by simply truncating the solid $[1,2]$. There are, two kinds of such differences, namely the surface relaxation and surface reconstruction, which have been studied extensively over the past few years. First is the rigid movement of one, or more, of the first surface layers in the perpendicular direction without of atomic rearrangement within the layers. The last surface phenomenon is associated with some specific phase transition in pure metals: atomic rearrangements within the outermost surface lattice with new 2D-symmetry. At present the clean metal surface known to reconstruct are the (100) faces of the single-crystal Pt, Ir, Au, Mo, W, V, Cr, the (110) faces of Pt, Ir, Au and the (111) face of Au [1]. It has been found that some surfaces reconstruct under the influence of adsorbates [3-6]. Reconstructed surface superstructures can also occur when clean metal surface has been prepared, spontaneously with temperature. Such specific surface phenomena take place also in the case of ordering alloy systems, for example, $\mathrm{Cu}-\mathrm{Au}, \mathrm{Cu}-\mathrm{Pt}, \mathrm{Pt}-\mathrm{Fe}, \mathrm{Co}-$ $\mathrm{Ni}, \mathrm{Pt}-\mathrm{Co}$ [2, 7-9]. The surface reconstruction phase transformation may be reversible or irreversible. It is very important for understanding the surface reconstruction mechanism and the nature of the surface phase stability to study the main characters phase transformation, the critical temperature, kinetics of surface reconstructions and the critical exponents.

Reconstructed low-index surfaces of noble metals (Pt, Ir, Au) have been of great interest to surface scientists ever since their discovery (1965). The particularly striking feature of the (100) and (110) faces of these metals is that the reconstructed phase appears to be stable configuration of these surfaces under clean conditions. This behaviour is different from that of other reconstructed metal surfaces, e.g. W(100), where a reversible structure transformation observed near $300 \mathrm{~K}$ [1].

The surface structure determination of catalytic active metals is of special interest. A detailed knowledge of the actual position of the catalyst surface atoms under certain pressure and temperature condition is of critical importance for the kinetics the reaction. Most of the pure metals and bimetallic catalysts used in the industry are based on noble metals [2, 7-9]. Last years the supported Pt-based catalysts are discussed [810].

It should be stressed that we have focused our attention in the present review only on the surface structure of well-defined low-index single crystals $\mathrm{Pt}, \mathrm{Ir}$ and $\mathrm{Au}$, because the kinetics of the adsorption and chemical reaction on pure metals can be understand on the basis of the kinetics 
parameters obtained from single-crystal studies. Many surface structure UHV analytical techniques have been used to investigate surface reconstruction. Basically, they can be categorized into reciprocal space techniques or diffraction techniques, as, e.g. low-energy electron diffraction (LEED), X-ray diffraction (XRD), and real-space techniques, as, e.g. lowenergy ion scattering (LEIS), scanning tunneling microscopy (STM). Bellow we present some important recent experimental and theoretical results in this direction.

\section{PHASE TRANSFORMATION OF THE (100) SURFACES}

\section{1. $\mathrm{Pt}(100)(1 \times 1)$ and $\mathrm{Pt}(100)-$ hex- $R$ Structures}

The clean $\mathrm{Pt}(100)$ surface is known to appear in two crystallographic phases, a metastable $(1 \times 1)$ structure and a reconstructed phase with a complicated LEED pattern [11]. It is commonly accepted that the latter is caused by a hexagonal close packing of the first atomic layer though the quantitative confirmation of this model by LEED structure determination is only at the beginning [11]. In most cases the superstructure was called $(5 \times 20)$ according to the size of the coincidence mesh. However this size is to some extent accidental and more reasonable characterization $\mathrm{Pt}(100)$-hex or $\mathrm{Pt}(100)$-hex- $R$ were used.

First detail observation of the kinetics structural transition $\mathrm{Pt}(100)(1 \times 1)$ $\rightarrow$ hex by LEED intensities was performed by Heinz et al. [12, 13]. They have shown that the structural transition of $\mathrm{Pt}(100)$ surface is irreversible. The $(1 \times 1)$ structure, which results by an ideal cut through the bulk reconstructs under temperature increase but is not restored by a following corresponding decrease. So the metastable bulk-like structure cannot be established by a simple temperature treatment, but is prepared by adsorption of atoms or molecules which can be carefully desorbed afterwards without structural change of the substrate.

The reorganization of surface atoms back to bulk structure during adsorption was interpreted by the fact that bulk layer chemical bonds are resorted causing the free energy of the surface to take its absolute minimum at the bulk $(1 \times 1)$ surface structure. This behaviour is demonstrated schematically in Fig. 1. The hexagonal superstructure is metastable at sufficiently high coverages and converts to the $(1 \times 1)$ structure by thermal activation. Careful desorption of the adlayer makes the surface return to the clean but metastable $(1 \times 1)$ phase.

The clean metastable $(1 \times 1)$ phase was prepared by intermediate NO adsorption by such way: the clean $\mathrm{Pt}(100)$-hex- $R$ surface was exposed to $\mathrm{NO}$ for $5 \mathrm{~min}$ at about $3 \times 10^{-8} \mathrm{mbar}$ and $350 \mathrm{~K}$ resulting in a streaked superstructure pattern. The streaks disappeared by heating the sample to about $420 \mathrm{~K}$ and $(1 \times 1)$ structure remains with, however, considerable background. This is believed to be due to disordered oxygen which was 


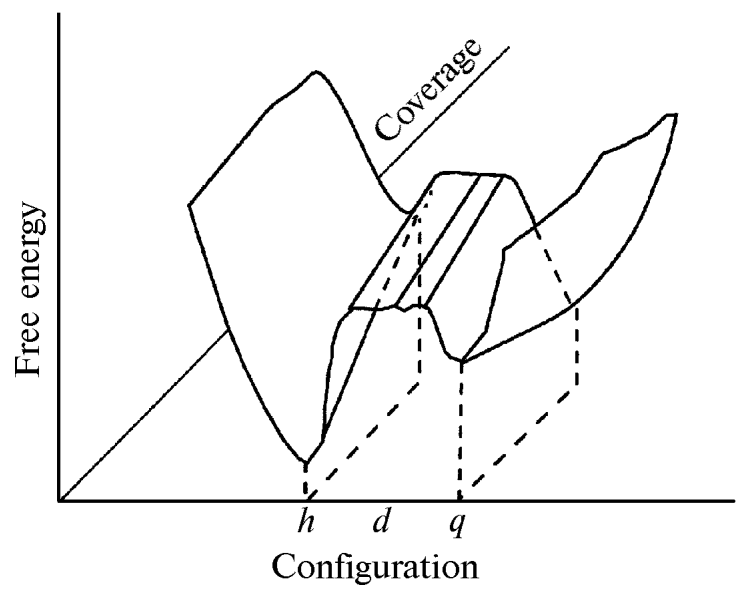

Figure 1. The changes of the free energy as a function of structural configuration and coverage (schematic) of the $\mathrm{Pt}(100)$ surface: $h$-hexagonal structure and $q-$ quadratic structure of the first layer; $d$-disordered state [12].

presumably produced by thermal dissociation of NO, while nitrogen desorbs. The reduction of oxygen in a hydrogen atmosphere of about $7 \times 10^{-8} \mathrm{mbar}$ for $5 \mathrm{~min}$ at $350 \mathrm{~K}$ has given a pure and background free $(1 \times 1)$ LEED pattern. In order to avoid residual $\mathrm{OH}$ molecules on the surface the sample was finally heated to $400 \mathrm{~K}$ but no further modification of the LEED pattern was observed.

The time and temperature dependencies of the integral intensities of both, of decreasing integer order spots as well as growing superstructure spots, were recorded using computer controlled TV method because the change of intensities takes place within the order of minutes down to some ten seconds depending on the actual temperature. For example pure $(1 \times 1)$ diffraction pattern, $(1 \times 5)$ superstructure spots develop within the order of a minute when the crystal temperature was increased to about $410 \mathrm{~K}$. The examples of intensity-temperature dependence and intensity-time dependence are presented in Fig. 2, 3. It appears that with increasing temperature, the intensity of the (10) spot decreases at first by simple diffuse scattering described by a Debye-Waller factor as demonstrated by the semi-logarithmic plot in Fig. $2 b(1)$. With a heating rate of about $2 \mathrm{~K} / \mathrm{s}$, the transition starts at about $425 \mathrm{~K}$ with a steep decrease of intensity and ends above $500 \mathrm{~K}$ where again a Debye-Waller like dependence begins. In the region of the transition, the intensity of the superstructure rises from zero to a maximum, which, however, is influenced by thermal-diffuse scattering dominating the following temperature range (Fig. 2c). Up to about $470 \mathrm{~K}$, the intermediate $(1 \times 5)$ structure shows up and only at higher temperatures the pattern splits into $(5 \times 20)$ or more precisely $(5 \times 25)$ or better $\mathrm{Pt}(100)$-hex. This latter structure is also me- 

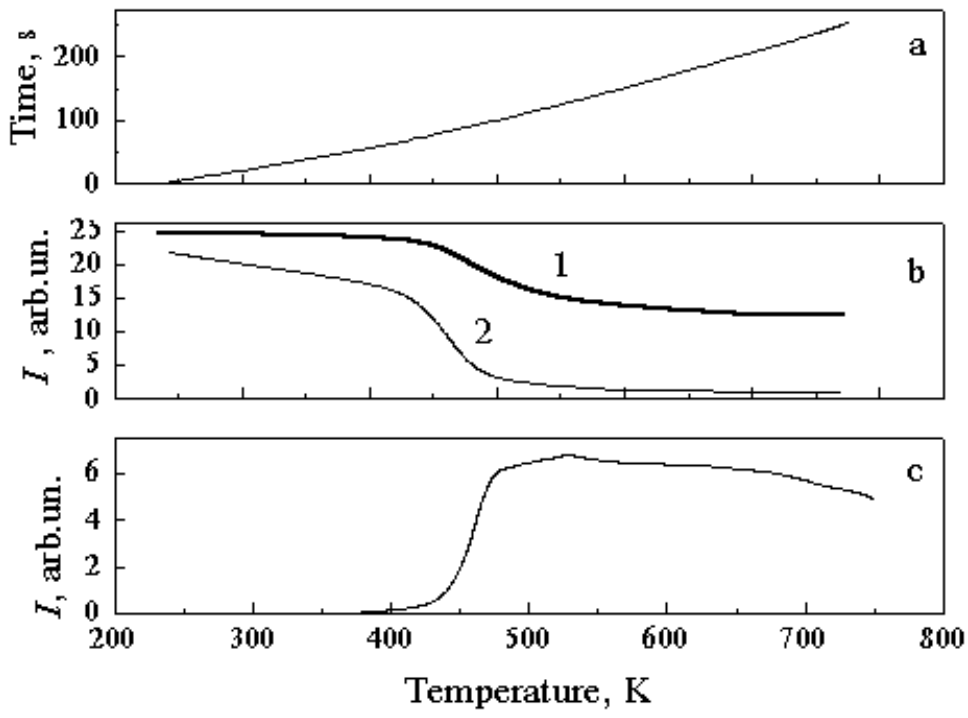

Figure 2. Intensity-temperature dependence at $E=85 \mathrm{eV}$ : a-time-temperature characteristic; b-intensity of integer order spot (10) (1-l, 2-In /); c-intensity of the superstructure spot (06/5) [12].

tastable and changes to $\mathrm{Pt}(100)$-hex- $R 0.7$ at above $1100 \mathrm{~K}$, a structure which corresponds to a $0.7^{\circ}$-rotation of the surface layer. It should be clearly pointed out that the metastability of the starting $(1 \times 1)$ phase forbids to give a precise transition temperature, which depends on the heating rate.

As demonstrated in Fig. 1, the transition $(1 \times 1) \rightarrow$ hex is believed to be an activated process. Heinz et al. [12] studied LEED intensities as a function of time (Fig. 3) for different but constant elevated temperatures as parameter and estimated the activation energy of the transition, $\Delta \AA=1.1$ $\mathrm{eV}$. In the next work Heinz et al. [13] examined in more detail LEED beam profiles and gave a geometrical model of the reconstruction based on the shift of atomic rows. According to this model the structure transition of the metastable $(1 \times 1)$ phase to the $(5 \times 25)$ structure takes place via a pure $(1 \times 5)$ structure with non-splitted but relatively broad $1 / 5$ order spots. This is due to the nucleation of quasi-hexagonally ordered domains of the first layer whose diameters were found to be about $100 \AA$ an average [12]. Quasi-hexagonally means, that the surface hexagon is in a way distorted to fit to the quadratic second layer, which mainly results in a unit mesh angle of about $59^{\circ}$ instead of the ideal $60^{\circ}$. When more and more such domains nucleate and grow during the process of the transition their edges come to meet each other. At elevated temperatures they from larger coherent hexagonally ordered areas. This reduces the half width of a superstructure spots as observed. The beam splitting can be 

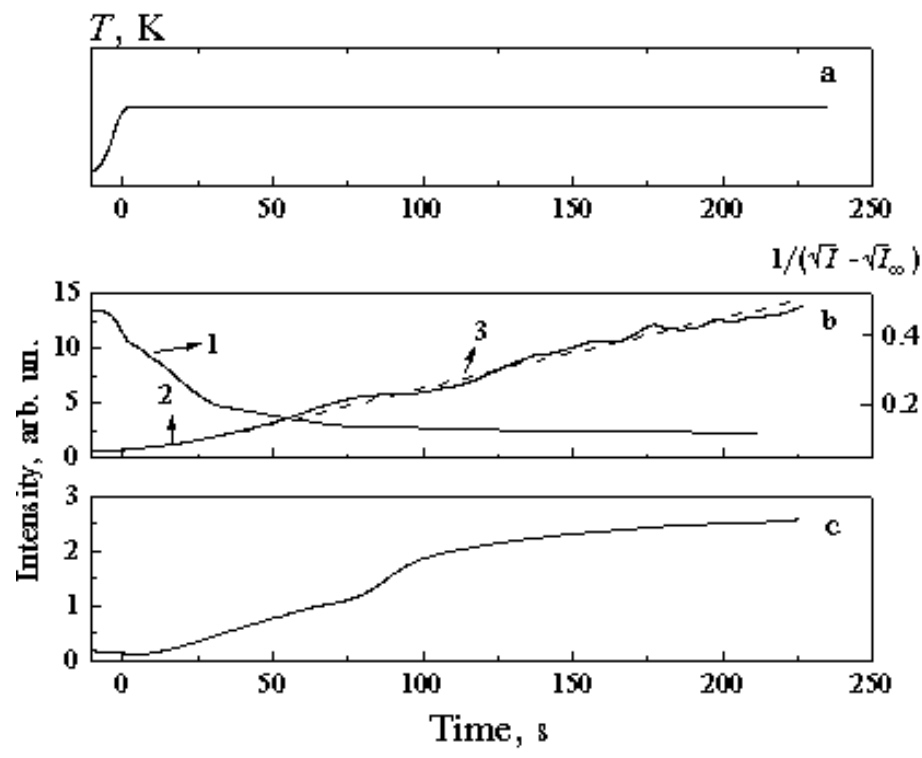

Figure 3. Intensity-time dependence at $413 \mathrm{~K}$ : a-time-temperature change behaviour; b-intensity of integer order; c-intensity of superstructure spots. $E_{0}=32$ eV [12].

interpreted by a simultaneous reordering of the surface atoms to give a nearly ideal unit mesh angle accompanied by a small compression of lateral atomic distances of about $4 \%$.

\section{2. $\operatorname{Ir}(100)(1 \times 1) \rightarrow(1 \times 5)$ Phase Transformation}

The reconstructed $\operatorname{Ir}(100)$ surface has a rather simple quasi-hexagonal superstructure $(1 \times 5)$ on the top layer. Several models have been proposed in order to explain the $(1 \times 5)$ LEED patterns such as the hexagonal model, the missing row hexagonal model, the shifted row model [11]. Among them, the buckled hexagonal model with bridge registry survives as the most realistic. The first study kinetics of $(1 \times 1) \rightarrow(1 \times 5)$ surface reconstruction on $\operatorname{Ir}(100)$ was presented by Heinz et al. [14]. Integrated LEED intensities and spot profiles were measured by means of a computer-controlled video system [12]. It is known that a clean $\operatorname{Ir}(100)$ surface has the reconstructed $(1 \times 5)$ phase which corresponds to thermodynamic equilibrium for the clean surface. In order to observe the transition $(1 \times 1) \rightarrow(1 \times 5)$ the undisturbed metastable $(1 \times 1)$ configuration must be reestablished. This was done by oxygen adsorption. On the reconstructed face adsorption of $\mathrm{O}_{2}$ at $5 \times 10^{-7}$ mbar was made at $475 \mathrm{~K}$ for about $2 \mathrm{~min}$. After heating to about $750 \mathrm{~K}$ and exposing to hydrogen at $550 \mathrm{~K}\left(5 \times 10^{-7}\right.$ mbar for about $1 \mathrm{~min})$ the LEED pattern was only $(1 \times 1)$. 
After having prepared to clean $(1 \times 1)$ phase as described, the transition $(1 \times 1) \rightarrow(1 \times 5)$ can be started by flashing to temperatures above $880 \mathrm{~K}$. The system overcomes the corresponding activation energy and reconstructs into the hexagonal close packed $(1 \times 5)$ structure. However the transition from a metastable to a stable state suffers from the fact that the system is far from thermal equilibrium in the beginning and approaches the equilibrium state only for long times. So the transition process is temperature and time controlled. This means that no transition temperature can be defined, the transition can not be described by giving a temperature dependence and it is irreversible. Dynamics of the thermal activation reconstruction process $\operatorname{Ir}(100)(1 \times 1) \rightarrow(1 \times 5)$ was investigated by measurement diffraction spot profiles as well integrated intensities as a function of time for different constant temperatures (Fig. 4, 5). It was shown that the transition starts by the formation of intensity streaks along the $(1 \times 1)$ unit mesh edges and superstructure spots develop from these streaks at the cost of integer-order spots. Both spot widths and intensities change rapidly in the beginning of the transition but subsequently vary very slowly without approaching their equilibrium value. This happens

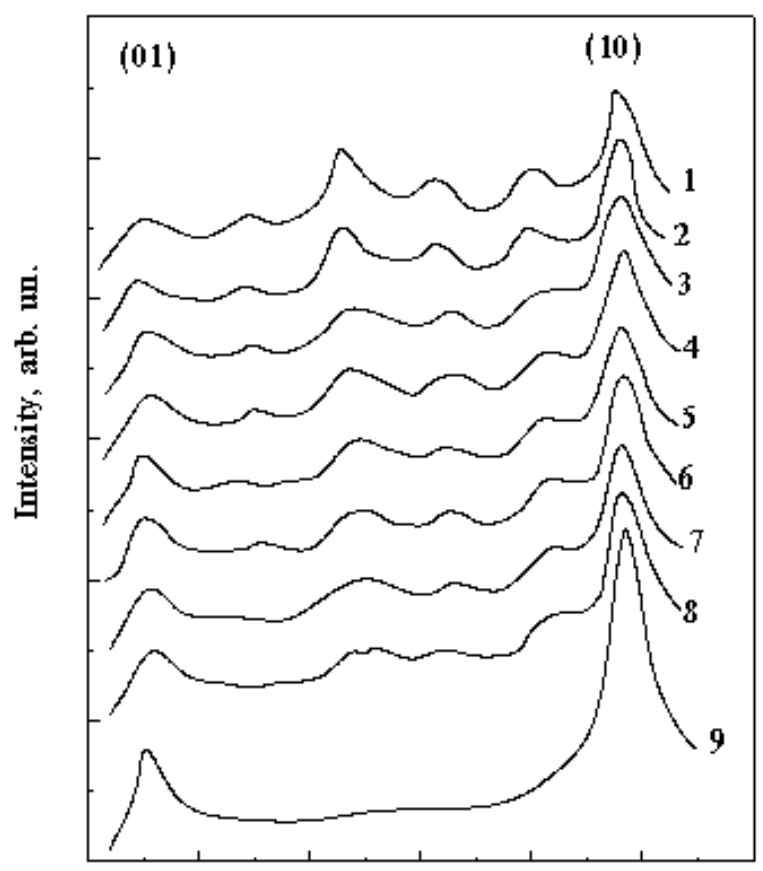

$\Delta k$, arb. un.

Figure 4. Diffraction spot profiles at $84 \mathrm{eV}$ for the (10) and (11) spots for several times after heating at $940 \mathrm{~K}$ : 1 -flesh $(1300 \mathrm{~K})$; 2-5 min; 3-2 min; 4-1 min; $5-30 \mathrm{~s} ; 6-20 \mathrm{~s} ; 7-10 \mathrm{~s} ; 8-5 \mathrm{~s} ; 9-(1 \times 1)$ structure [14]. 


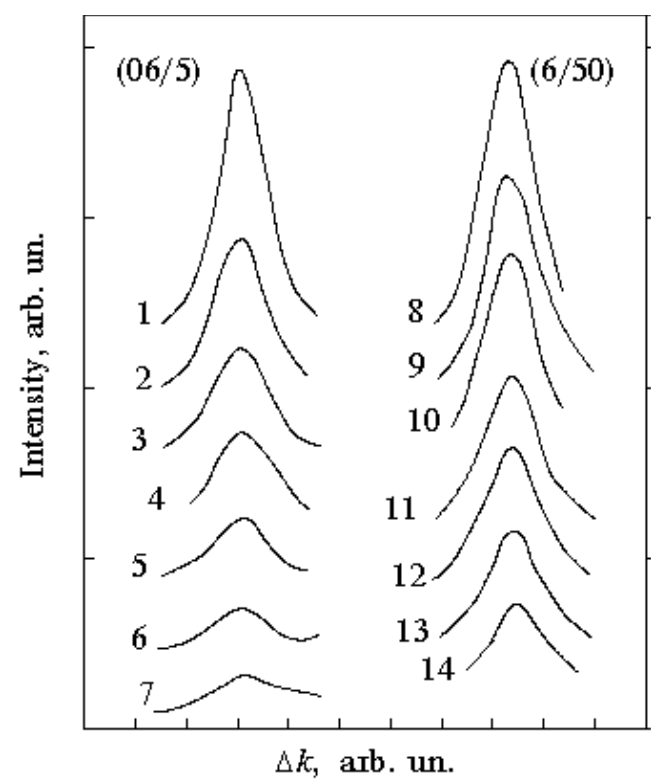

Figure 5. The change of the $(06 / 5)$ and $(6 / 50)$ spot profiles. $E=99 \mathrm{eV} ; T==960$ $\mathrm{K}[14]$.

only after a flash to near or above $1300 \mathrm{~K}$. The development of intensity streaks along the $(1 \times 1)$ unit mesh edges indicates that at least partial disorder appears in the beginning of the transition. The streaks are interpreted to correspond to linear atomic rows, which have no phase correlation. The transition is assumed to start from the ordered phase via shifting of atomic rows forming a nearly hexagonal unit mesh. Of course the final quasi-hexagonally close-packed structure shows a higher density of surface atoms that the initial $(1 \times 1)$ phase. Therefore the formation of steps must be the consequence. The activation energy of the transition was determined from the intensity increase to be $0.88 \pm 0.03 \mathrm{eV}$.

\subsection{Structures and Phases of the $\mathrm{Au}(100)$ Face}

Like the (100) faces of $\mathrm{Pt}$ and Ir, the $\mathrm{Au}(100)$ surface layer is believed to be hexagonal at room temperature in contrast to the square symmetry of the bulk lattice planes lying immediately beneath.

Gibbs et al. [15] have presented the most detailed study of the structure and phases of the clean $\mathrm{Au}(100)$ surface between room temperature and the bulk melting temperature of $1337 \mathrm{~K}$. These experiments were performed in the glancing-incidence geometry, using an UHV apparatus for X-ray surface scattering. They identified three distinct structural phases exhibited by the $\mathrm{Au}(001)$ surface between $T=300 \mathrm{~K}$ and $T=1337$ 


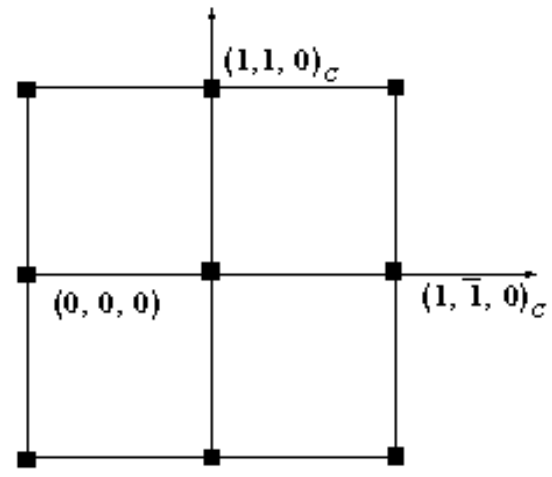

Figure 6. LEED pattern of the disordered phase [15].

$\mathrm{K}$. They are (1) disordered $(1170<T<1320 \mathrm{~K})$, (2) distorted-hexagonal $(970<T<1170 \mathrm{~K})$, and $(3)$ rotated, distorted-hexagonal $(300<T<970)$. The diffraction pattern observed in the high-temperature phase between $T=1170$ and $1337 \mathrm{~K}$ is shown in (Fig. 6). Solid squares are indexed in bulk-cubic reciprocal-lattice units $(h, k, I)$. The area enclosed by the solid squares corresponds to the substrate unit cell.

At elevated temperatures, the diffraction pattern has the $(1 \times 1)$ symmetry of the bulk, consistent with an unreconstructed or disordered surface layer. Below $T=1170 \mathrm{~K}$, there is a reversible transition to an incommensurate, two-dimensional structure of hexagonal symmetry. Hexagonal reciprocal-lattice vectors $(1,0) h$ and $(0,1) h$ are indicated by open triangles in Fig. 7. Surrounding each are additional satellites along directions parallel to the bulk [110] direction; these are indicated by solid circles. The sepa-

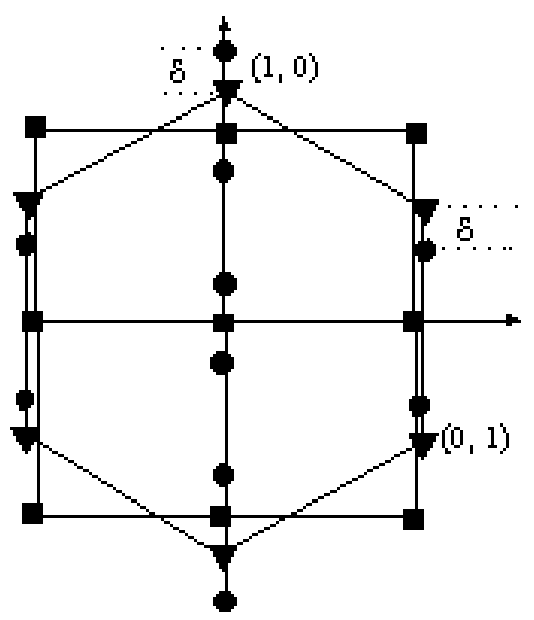

Figure 7. LEED pattern of the disordered-hexagonal phase [15]. 


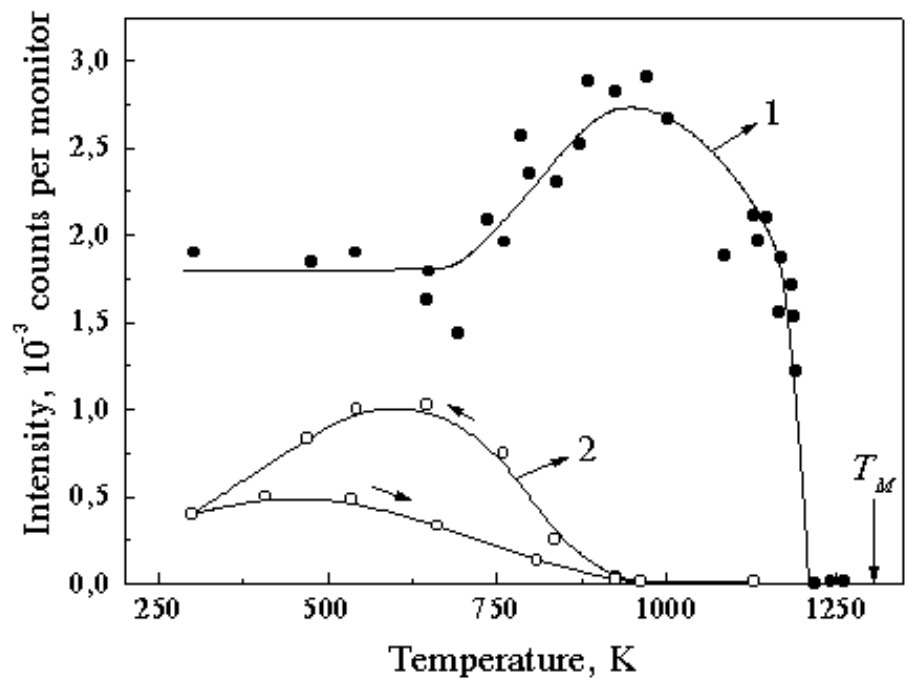

Figure 8. Intensity-temperature dependence of the non-rotated (1) and rotated (2) components of the hexagonal peak [15].

ration between harmonics is the incommensurability $\delta=0.206$ ( \pm $0.001) \sqrt{2} c^{*}\left(c^{*}=2 \delta / c=3.081 \AA^{-1}\right.$ at room temperature $)$. Below $T=970 \mathrm{~K}$, additional roads appear around each of the hexagonal rods. These occur at a fixed angle equal to $\pm 0.81^{\circ}$ away from the [110] direction, and reveal the existence of rotated domains $(5 \times 1)$. The transition to the rotated, distorted-hexagonal phase is reversible. Gibbs et al. [15] also discussed the temperature dependence of the diffraction pattern obtained for the $\mathrm{Au}(100)$ surface. For example, the integrated intensities of the rotated and non-rotated components of the hexagonal peak at $(1,0) h$ is plotted in Fig. 8. An abrupt increase in intensity for temperatures decreasing from $T$ $=1200 \mathrm{~K}$ is seen clearly. The width of the transition is about $10 \mathrm{~K}$. It was found that both the incommensurability and the average rotational angle were weakly temperature dependent.

\section{SURFACES WITH (110) ORIENTATION}

\section{1. $\mathrm{Au}(110)(1 \times 1) \Leftrightarrow(1 \times 2)$ Phase Transformation}

At room temperature the (110) surfaces of $\mathrm{Au}, \mathrm{Pt}$, and Ir reconstruct to a structure with $(1 \times 2)$ symmetry. It was shown above that a variety of experiments and total energy calculations clearly indicate that the $(1 \times 2)$ structure, of this metals is a missing-row (MR) geometry (Fig. 9). It is seen from this figure the ideal (110) surface represents a set of parallel atomic rows and every second row is missing in the $(1 \times 2)$ phase. Note 
(110)

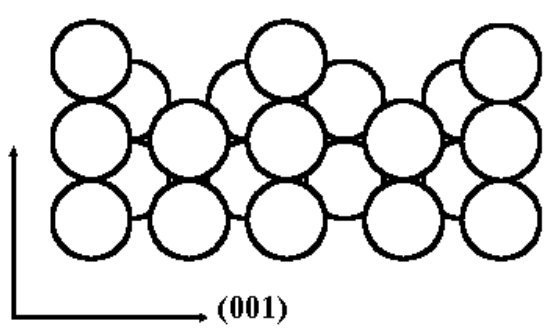

Figure 9. Cross section of the missing row structure model.

that the MR model is highly anisotropic. The attraction along the [1 10$]$ rows is rather strong, and the net repulsion between adjacent rows is weaker by more that a factor of 10 [16]. At some temperature $T_{c}$ a reconstructed $(1 \times 2)$ surface undergoes a reversible 'deconstruction' to a high temperature $(1 \times 1)$ structure which is no longer reconstructed. The nature of this phase transition has been studied with variety of experimental and theoretical approaches. The main results of this studies were performed for $\mathrm{Au}(110)(1 \times 2) \Leftrightarrow(1 \times 1)$ deconstruction transition.

Wolf et al. [17] first investigated the dependence of the integrated intensity of the LEED $(0,1 / 2)$ reflex as a function of the temperature and have found that a continuous phase a transition from $(1 \times 2)$ superstructure to the normal $(1 \times 1)$ structure take place at $713 \mathrm{~K}$. Campuzano et al. $[18,19]$ have shown that this $(1 \times 2) \Leftrightarrow(1 \times 1)$ structural change is an orderdisorder phase transition and that the $(1 \times 1)$ pattern seen in LEED belongs to a disordered top layer on an ordered second layer in the lattice gas sense. These authors reported a transition temperature at $T_{c}=650 \mathrm{~K}$ for the $\mathrm{Au}(110)$ surface. They used high resolution LEED systems to a detailed study the line shape and integrated intensity as a function of temperature and reported first measurements of the critical exponents of this continuing phase transition. The results show that the $\mathrm{Au}(110)(1 \times 2) \Leftrightarrow(1 \times 1)$ transition is strictly two-dimensional, belonging to the Ising universality class with the exponents $\beta=0.13 \pm(0.022), \gamma=1.75$ \pm 0.03 , and $v=1.02 \pm 0.02$ (the predicted values are $0.125,1.75$ and 1 , respectively). This was earlier predicted by Bak [20] on the basis of symmetry considerations. It has been suggested by Campuzano et al. [19] that the high temperature $(1 \times 1)$ structure is disordered. The transition from ordered missing-row structure to disordered does not involve any mass diffusion, because on average only half monolayers of $\mathrm{Au}$ 'adatoms' a top on $\mathrm{Au}(110)$ surface one lattice spacing. It is suggested that the missing-row structure is formed as atoms in terraces on the surface migrate the first time the crystal is heated. According to this view, the $(1 \times 2)$ regions on the surface would have finite sizes, the average size of the terrace widths. On the base of analysis the beam profiles of the (1, $-1 / 2$ ) spots they determined the average size of ordered regions to be $150 \AA$. 
Clark et al. [21] have extracted the specific-heat critical exponent $\alpha$ for the $\mathrm{Au}(110)(1 \times 2)$ order-disorder phase transition using integrated LEED intensities. The resulting value $\alpha=0.02 \pm 0.05$ is consistent with the predicted Ising universality class $(\alpha=0)$ of this transition. They also estimated the critical exponent $v=1.1 \pm 0.1$ from the measured increase in the FWHM of the $(0,-1 / 2)$ beam in the range $0.02<t<0.07(t=(T$ $\left.-T_{c}\right) / T_{c}$ is the reduced temperature). This result agrees with the Ising value $v=1$. These values for $\alpha$ and $v$ rule out the possibility that the transition is first order concluded Clark et al. They also obtained the critical temperature $T_{c}=695 \pm 3 \mathrm{~K}$ which is substantially larger than the $650 \mathrm{~K}$ [19]. Crark et al. concluded that this difference is consistent with that expected from finite-size effects. The effective critical temperature $T_{e}$ is expected to vary with the scale $L$ of finite-size regions approximately as

$$
T_{c}-T_{e} \equiv \Delta T \cong a L^{v} T_{c},
$$

where $a$ is a constant and $L$ is measured in lattice spacing $[0.408 \mathrm{~nm}$ along the [110] direction for $\mathrm{Au}(110)]$.

McRae [22] at latest of the studies, attributes this variability to trace impurities and find $T_{c}=765 \mathrm{~K}$. They investigated the segregation of $\mathrm{Sn}$ to the (110) surface of a nominally pure Au crystal by LEED, AES, and lowenergy ion scattering. Sn coverages up to 0.2 monolayer were produced by annealing above $675 \mathrm{~K}$. Surface Sn was shown to have a marked effect on the $(1 \times 2) \Leftrightarrow(1 \times 1)$ phase transition, e.g. Sn coverages of 0.002 and 0.13 monolayer produced shifts to lower transition temperatures of 15 and $200 \mathrm{~K}$, respectively.

Derks et al. [23] reported first results of a near order analysis of the $\mathrm{Au}(110)$ surface using low energy ion $\left(\mathrm{K}^{+}, 300 \mathrm{eV}\right)$ scattering and in the a computer based data analysis in the temperature range of $300-800 \mathrm{~K}$. The results showed (i) that the top layer atoms at $800 \mathrm{~K}$ remain in their regular lattice sites, as suggested previously [18, 19], (ii) the atoms occupy available lattice sites at random, and (iii) the random layer contains as many atoms as the $(1 \times 2)$ top-layer, i.e. 1/2 monolayer (Figs. 10a and $10 \mathrm{~b})$. The near order structure gives the necessary information for a microscopic understanding of the phase transition and may answer the question whether large mass transport is occurring during the phase transition. Derks et al. [23] concluded that considering the LEED results $[18,19]$ the case of the 'random' $1 / 2$ monolayer may be more likely, which requires that at most $1 / 4$ of monolayer has to be moved by one $<001>$ lattice distance during the phase transition. This interpretation of the ion scattering results establishes the hypothesis that the $\mathrm{Au}(110)$ surface has at all temperature an 'absorbed' layer of Au atoms corresponding to $1 / 2$ of the full layer. At high temperature $(800 \mathrm{~K})$ the adatoms occupy regular lattice sites randomly and form a partly ordered $(1 \times 2)$ structure at low temperatures. Their it is implicitly assumed that the phase transition occurs between a full $(1 \times 1)$ surface to the $(1 \times 2)$ surface with an 


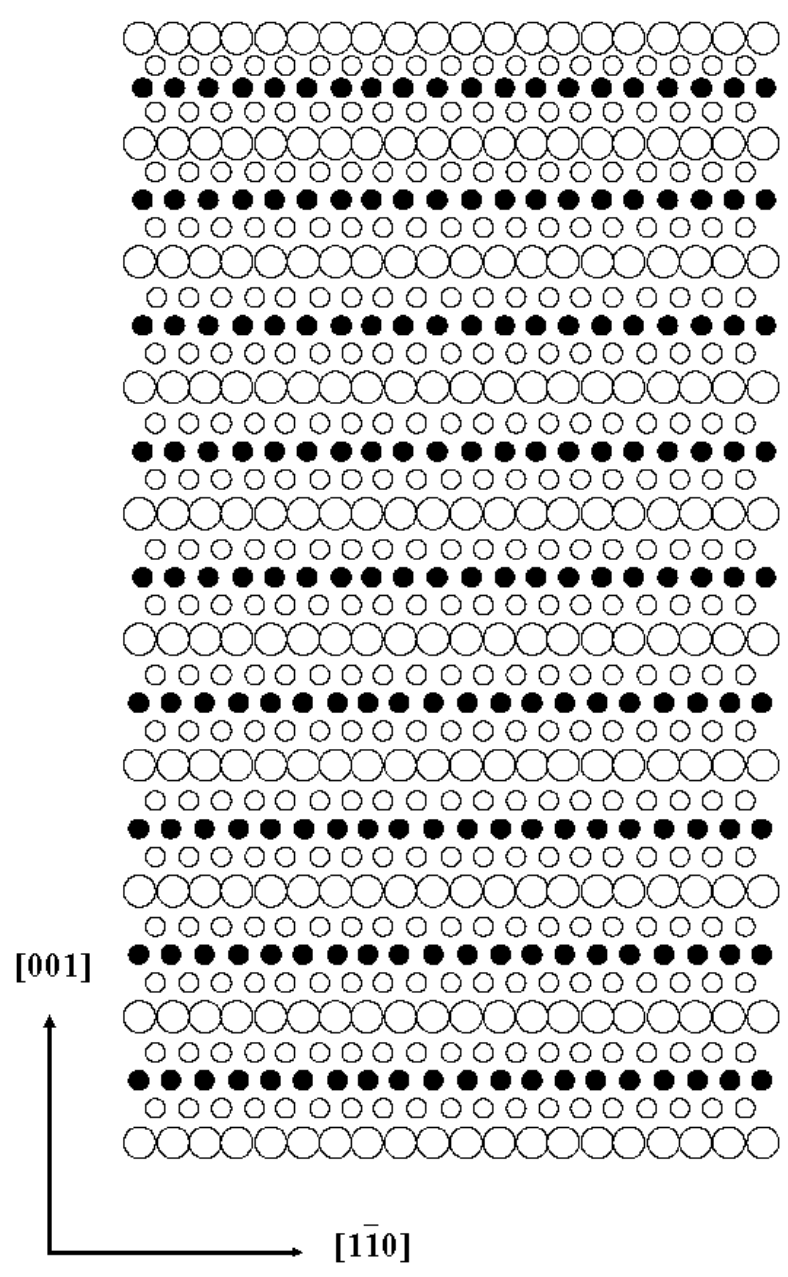

Figure 10a. Schematic f.c.c. (110) surface-the ideal $(1 \times 2)$ reconstruction. Large circles $-1^{\text {st }}$ layer, small circles- $2^{\text {nd }}$ layer, black dots $-3^{\text {rd }}$ layer [23].

occupancy of $1 / 2$ monolayer. The authors agreed with the assumption of

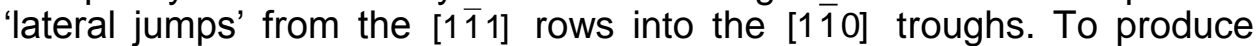
Figs. 10a and 10b, correlated jumps, as proposed in Ref. [24], are not necessary. Pairs are formed accidentally, pairs and longer chains may be formed in addition due to the thermal diffusion along the rows.

Dückers and Bonzel [25] have measured $T_{c}$ using surface core-level shifts spectroscopy. The core-level binding energy separation between surface and bulk atoms, as well as the intensity ratio of surface-to-bulk contributions in a photoemission spectrum strongly depend on the structure of surface.

Many $4 f_{1 / 2}$ spectra of the clean $\mathrm{Au}(110)$ face for temperature between 


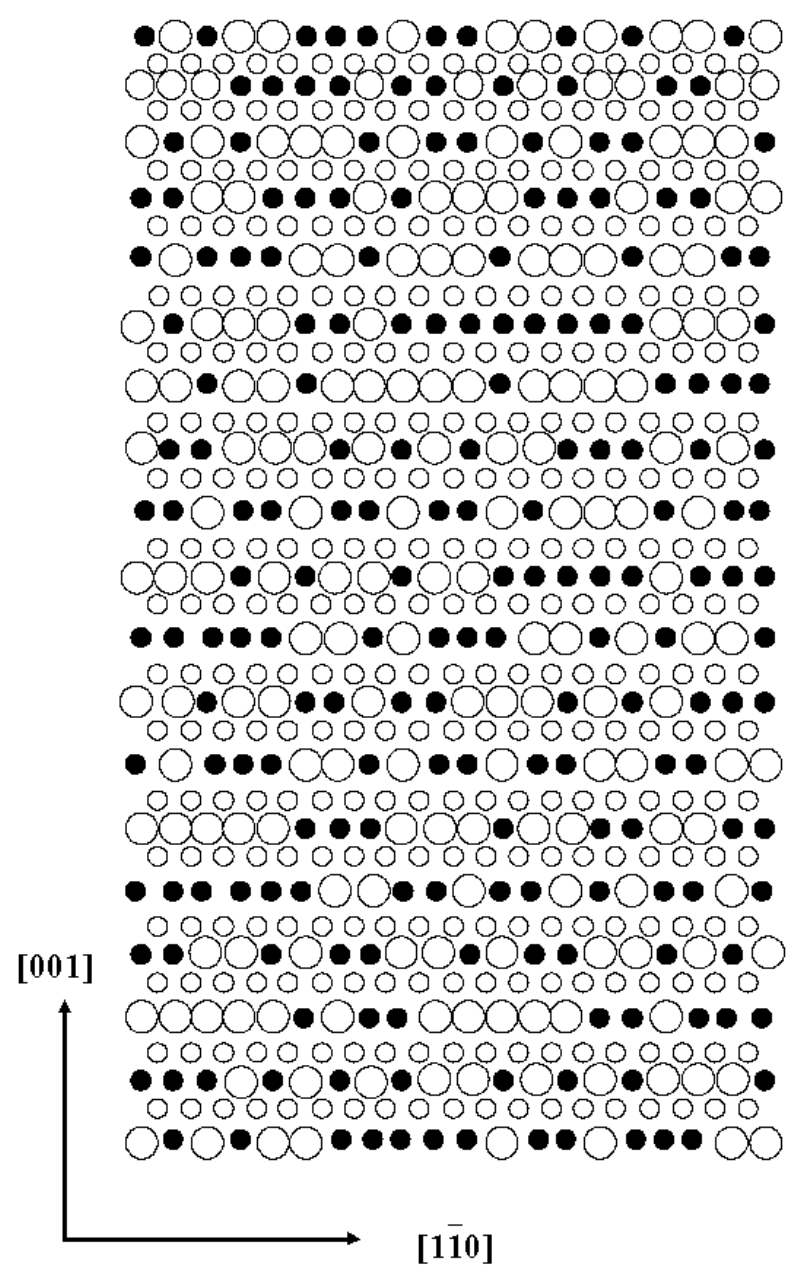

Figure 10b. Schematic f.c.c. (110) surface-the $(1 \times 1)$ surface with a random $1 / 2$ monolayer adsorbed. Large circles $-1^{\text {st }}$ layer, small circles-2 $2^{\text {nd }}$ layer, black dots-3 $3^{\text {rd }}$ layer [23].

$100 \mathrm{~K}$ and $850 \mathrm{~K}$ were measured. As can be seen from Fig. 11 values for the core-level shift $\Delta_{s, b}$ are nearly constant for temperatures below $500 \mathrm{~K}$ and above $750 \mathrm{~K}$ but in region $500 \mathrm{~K}<T<750 \mathrm{~K}$ they show a clear decrease. This behaviour in Fig. 11 indicates a change of the $\mathrm{Au}(110)$ surface structure between $500 \mathrm{~K}$ and $750 \mathrm{~K}$. Dückers and Bonzel concluded that this structure change corresponds to the order-disorder transition of $\mathrm{Au}(110)$ from the $(1 \times 2)$ to the disordered $(1 \times 1)$ surface. From Fig. 11 they find a transition temperature of $T_{c}=620 \pm 50 \mathrm{~K}$.

Recently van de Riet et al. [26] have reported ion scattering experiments from the $\mathrm{Au}(110)$ surface in the temperature range $300 \mathrm{~K}$ to $900 \mathrm{~K}$ 


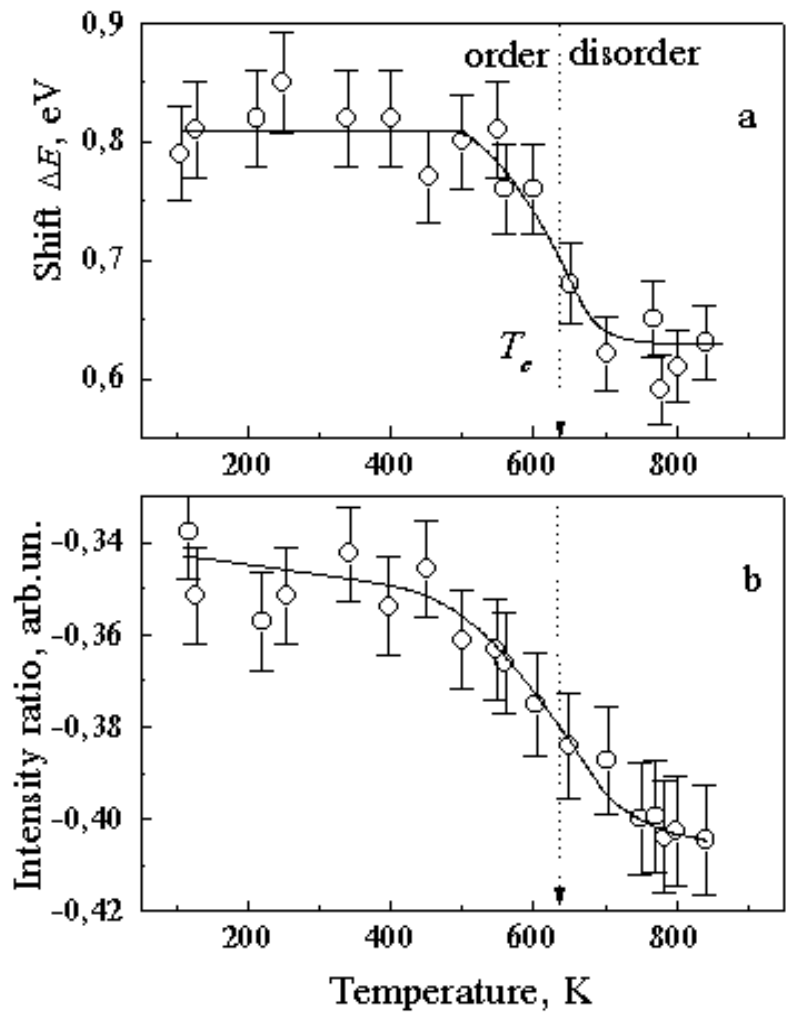

Figure 11. Energy shift (a) and intensity ration (b) for the clean $A u(110)$ surface as function of temperature [25].

and made some conclusion on the surface structure in the [110] and [001] azimuth. They measured polar angular ion spectra of $4.6 \mathrm{keV}$ neon ions scattered over $120^{\circ}$ and compared them to calculated spectra. It was found that below $530 \mathrm{~K}\left(120 \mathrm{~K}\right.$ below $\left.T_{c}=650 \mathrm{~K}\right)$ the chains in the [110] direction have no vacancies, whereas above this temperature the relative number of vacancies was a strongly increasing function of the temperature. Above the $(1 \times 2)-(1 \times 1)$ transition temperature the order in the [110] direction breaks down and spectra indicated the formation of steps above $T_{c}$. The spectra measured in the [001] direction indicate that the $(1 \times 2)-$ $(1 \times 1)$ transition is an order-disorder transition. The activation energy of vacancy formation was estimated to be $0.2 \mathrm{eV}$.

Most recently, Keane et al. [27] have presented the results of a synchrotron X-ray scattering study of the thermal disordering of the $\mathrm{Au}$ $(110)-(1 \times 2)$ reconstructed surface. In this study $(1 \times 2)$ surface domains extended $550 \AA$ in the [001] direction and $1630 \AA$ in the [110] direction, which are significantly large than those reported in previous studies [19]. Observing the temperature dependence of the superlattice and integral- 
order bulk-forbidden (anti-Bragg) surface peaks, as well as the specular (110) reflectivity they found that at $T_{c}=735 \mathrm{~K}$ the $(1 \times 2)$ surface undergoes a deconstruction transition characterized by the proliferation of compact anti-phase defects, with no measurable increase in the density of surface steps. It was found that this transition is described by critical exponents $(\beta=0.114, \gamma=1.45 \pm 0.15, v=0.75 \pm 0.1)$ close to those characterizing a two-dimensional Ising transition. Keane et al. [27] also found that by $784 \mathrm{~K}$ there has been a significant increase in the densities of both anti-phase defects and surface steps of the type associated with surface roughening. This suggests that the $\mathrm{Au}(110)$ surface is disordered in a two-step process, deconstruction followed by roughening, with a difference in the two transition temperatures of less that $50 \mathrm{~K}$.

\subsection{Surface Reconstruction of the $\mathrm{Pt}(110)$ Face}

The surface of $\mathrm{Pt}(110)$ has the same $(1 \times 2)$ missing row reconstruction as $\mathrm{Au}(110)$. The experimental observation of a similar transition for $\mathrm{Pt}(110)$ is not so clear as for $A u(110)$ one. The fist attempt of Salmeron and Somorjai [44] to observe $\operatorname{Pt}(1 \times 2) \Leftrightarrow(110)$ transition with LEED was not successful. They reported that the $(1 \times 2)$ LEED spot intensity versus temperature showed features at 520, 720, 920, 990, and $1040 \mathrm{~K}$. Above the last temperature, the $(1 \times 2)$ superspot disappeared entirely. However, the authors some concern about oxygen contamination.

Recently Dückers and Bonzel [25] have performed an attempt to determine the transition temperature for $\mathrm{Pt}(110)$ using the temperature dependence of surface core-level shifts. Fig. 12 shows the variation of shift $\Delta_{s, b}$ and intensity ratio $I_{s} I_{b}$ for the $\mathrm{Pt}(110)$ surface as a function of temperature. Thus all features observed for the order-disorder $(1 \times 2) \Leftrightarrow(1 \times 1)$ transition on $\mathrm{Au}(110)$ (Fig. 11) are reproduced also for Pt(110), except for a shift of the transition to higher temperature. Unfortunately the Pt surface did not stay clean at all temperatures. In the region $850 \mathrm{~K}<T<1050$ $\mathrm{K}$ the authors observed potassium corresponding to a coverage of 0.02 on the surface. They explain the experimental results as indication for an order-disorder transition for the $\operatorname{Pt}(110)$ surface at $940 \pm 50 \mathrm{~K}$. Only most recently two detailed experimental studies the clean $\mathrm{Pt}(110)$ reconstructed $(1 \times 2) \Leftrightarrow(1 \times 1)$ phase transition and its critical phenomena were reported [28, 29]. Robinson et al. [28] reported X-ray diffraction results for the $\mathrm{Pt}(110)$ phase transition, which they observed at $T_{c}=1080 \pm 50 \mathrm{~K}$. In many ways they found similar behaviour to $\mathrm{Au}(110)$ [19], with one important difference. Above $T_{c}$ steps are created spontaneously and their density diverges with temperature. This demonstrates conclusively that $\mathrm{Pt}(110)$ roughens above $T_{c}$. The simultaneous involvement of steps and reconstruction means that the ground state is at least fourfold degenerate and so the phase transition can no longer be classed as an Ising model. Yet the critical exponents $\beta=0.11$ and $v=0.95$ are consistent with the 

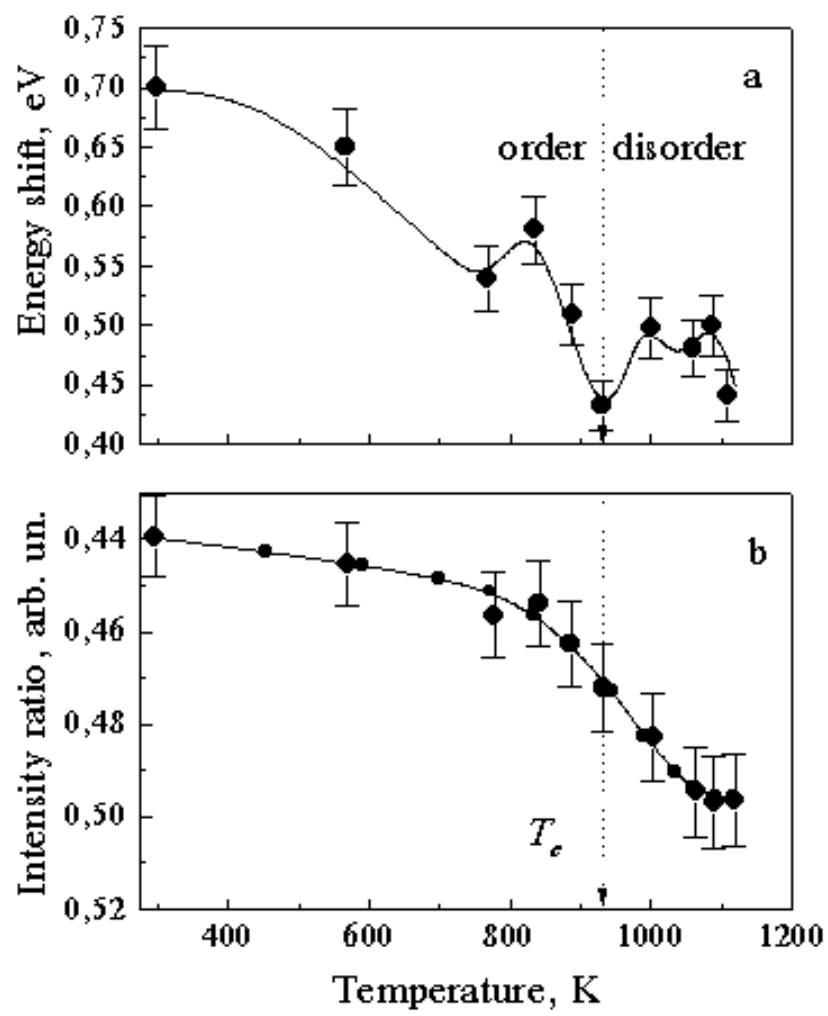

Figure 12. Shift (a) and intensity ration (b) as a function of temperature for Pt(110) surface [25].

Ising 2D-model, and agree well with $\mathrm{Au}(110)$ [18] (although those measurements were insensitive to steps). Thus the transition $\mathrm{Pt}(110)$ $(1 \times 2) \rightarrow(1 \times 1)$ must be classified as a roughening transition and not a simple two-state Ising 2D-transition. The temperature dependence peak height, peak shift and half-width are shown, for example in Fig. 13.

Zuo et al. [29] have presented a high-resolution LEED study of the clean $\mathrm{Pt}(110)$ reconstructed $(1 \times 2)$-to- $(1 \times 1)$ phase transition and its critical phenomena. The HRLEED (or spot-profile analysis LEED) has a resolution of $\sim 6 \times 10^{-3} \AA^{-1}$ in $\mathbf{k}$-space, about a factor of 10 better than the resolution of conventional LEED. Such a resolution allows to accurately extract the $(1 \times 2)$ structure factor

$$
\mathbf{S}(\mathbf{k}, T)=I_{0}(T) f(\mathbf{k})+\chi(\mathbf{k}, T)
$$

from the half-order beams over a wide range of $\mathbf{k}$, so that the critical exponents which are related to $\mathbf{S}(\mathbf{k}, T)$ can be accurately determined. For the Ising model in the critical scattering region 


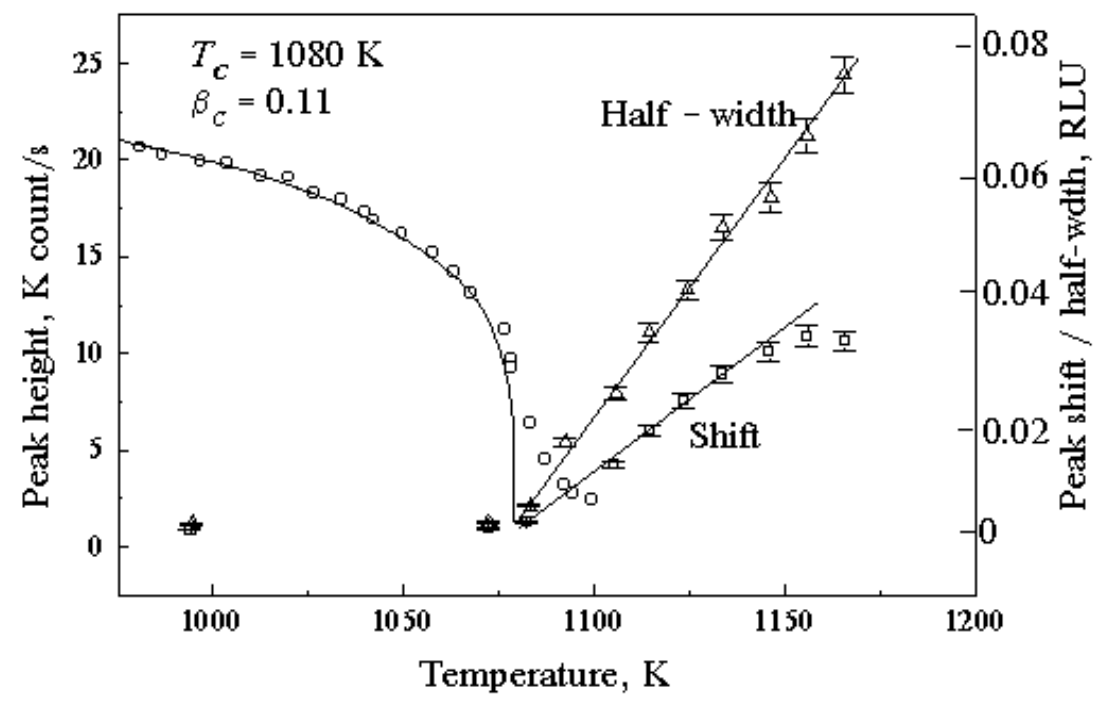

Figure 13. Temperature-dependent peak height and its fit by $\left(T_{c}-T\right)^{2 \beta C}$ (left), and peak shift (right) [28].

$$
\begin{gathered}
I_{0}(T) \sim t^{2 \beta}, \\
\chi_{0}(T) \sim(-t)^{-\gamma} \sim k^{\eta-2}, \\
k(T) \sim(-t)^{v},
\end{gathered}
$$

where $t=1-T / T_{c}$ is the reduced temperature and $\beta, \gamma$, and $v$ are the critical exponents. Those critical exponents describe the decay of the longrange order, the fluctuation of the short-range order, and the decay of the correlation length of the order parameter, respectively. The critical exponents only depend on the symmetry of a system, but not on the detailed interaction energies among atoms. Therefore, various systems can be grouped into a few universality classes. Therefore, a measure of the structure factor $\mathbf{S}(\mathbf{k}, T)$ from a superlattice beam near $T_{c}$ allows to determine the critical exponents $\beta, \gamma$, and $v$, as well as to test the scaling relation $\gamma=(2-\eta) v$. The theoretical predictions for $\beta, v, \gamma$ and $\eta$ of the Ising 2D-model are $0.125,1,1.72$, and 0.25 , respectively.

Temperature-dependent Bragg peak intensities $I(T)$ of the $(0,1 / 2)$ beam are shown in Fig. 14 for $E=85.69$ and $40 \mathrm{eV}$. These $I(T)$ curves were reversible in temperature and a rapid decay of the Bragg peak intensity occurs around $T_{c}=960 \mathrm{~K}$. The Bragg peak intensity of a superlattice beam versus temperature is a measure of the square of the order parameter at $T<T_{c}$ and is a measure of the fluctuation of short-range order at $T>T_{c}$. The broadening of the $\mathrm{FWHM}_{s}$ begins at almost the same temperature, $\sim 960 \mathrm{~K}$, as the drop in peak intensity. The continuous drop 


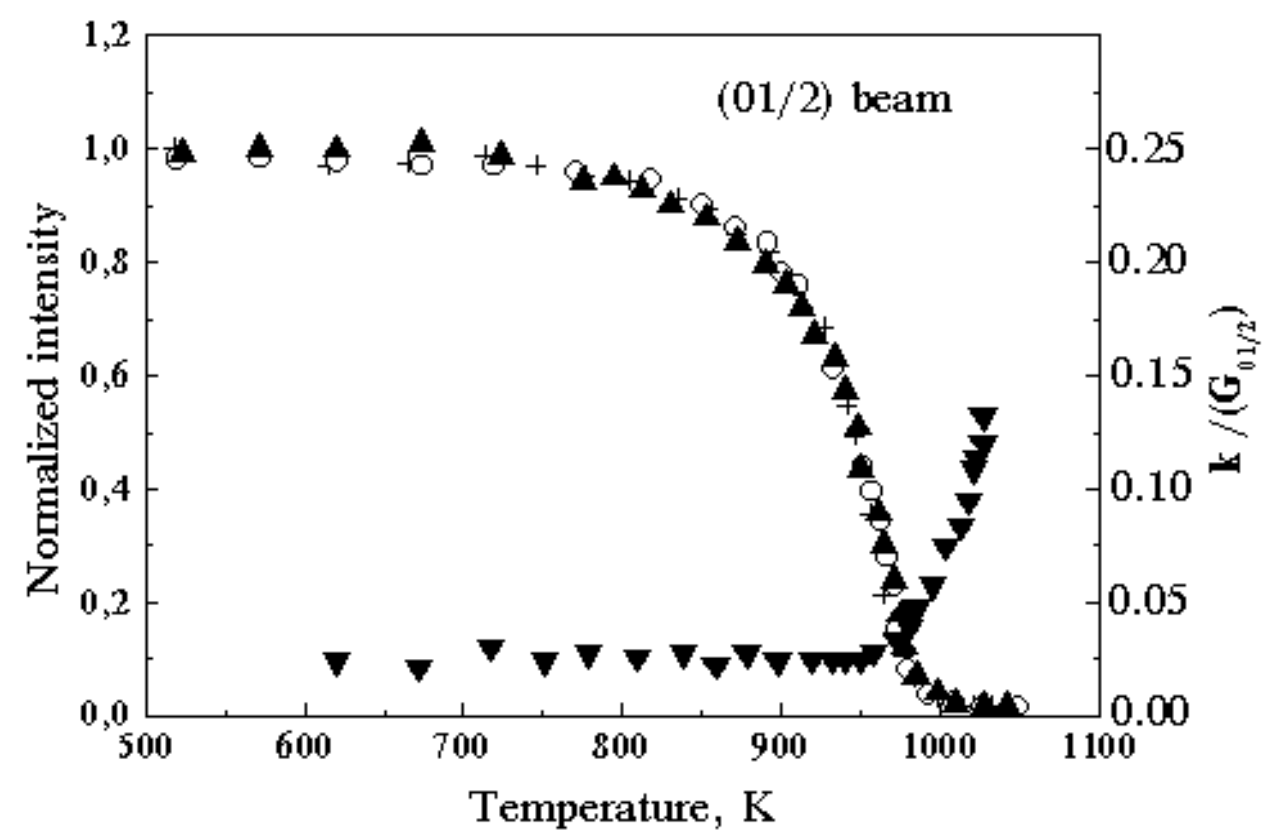

Figure 14. Temperature-dependent of the peak intensity of the (01/2) beam; $E=$ 85,69 , and $40 \mathrm{eV}$. The FWHMs is shown in the right hand scale. $\mathrm{G}_{(01 / 2)}$ is the magnitude of the superlattice reciprocal lattice vector spanned from the $(00)$ beam to the $(01 / 2)$ beam [29].

of the $(01 / 2)$ beam peak intensity, the continuous broadening of the FWHM, the absence of qualitative changes in the $I(E)$ profiles, all as a function of temperature, clearly indicate that the $(1 \times 2)$ surface-layer disorders around $960 \mathrm{~K}$ and the transition is of second order. The anisotropic broadening in the [110] and [001] directions Zuo et al. [29] explained by the persistence of the short-range order in the form of short chains of atoms along the [1 $\overline{1} 0]$ direction with little co-ordination among the rows.

In the critical region the physical quantities $I_{0}(T), \chi_{0}(T), k(T)$, which describe the critical phenomena, the authors used an asymptotic power laws of the from $A_{n}|t|^{n}(n=2 \beta,-\gamma, v)$. The critical exponents $(\beta=0.125, \gamma=$ $1.74, v=0.99)$ extracted from the $(01 / 2)$ superlattice beam were in excellent agreement with the prediction of the Ising 2D-class. However, an indication of increasing step density accompanying with the Ising transition was also observed. It was found also that the observed critical scattering maximizes around $T_{c}$ and was limited by the finite size of the $(1 \times 2)$ ordered region. At $T>T_{c}$, where only the short-range ordering exists, the structure factor of the superlattice, represented by $(01 / 2)$ beam, showed scaling and isotropic behaviour even though the broadening along the [110] and the [001] directions was anisotropic. 


\subsection{Theoretical Studies}

Some theoretical studies were also devoted to the problems of the (110) surface f.c.c. crystals. Bak [20] fist has proposed a physical realization of Ising 2D-model to describe critical behaviour for $(2 \times 1) \Leftrightarrow(1 \times 1)$ transition. The temperature dependence of the order parameter, the intensities of the Bragg-satellites should go as

$$
I \sim\left(T_{c}-T\right)^{2 \beta}, \beta=1 / 8
$$

The exponents $v, \gamma$, and $\eta$ can be studied by measuring the critical scattering near $T_{c}$. This idea was first supported by high-resolution LEED experiments [18].

Foiles and Daw [31] found in previous calculations with the embeddedatom method (EAM) that the missing-row structure is indeed lower in energy than the $(1 \times 1)$ structure for both $\mathrm{Pt}$ and $\mathrm{Au}(110)$ surfaces. In the next work Daw and Foiles [32] have shown that Monte Carlo simulations using energies computed via the EAM show the occurrence of orderdisorder transition on $\mathrm{Au}$ and $\mathrm{Pt}(110)$. They presented calculations determine the equilibrium structure as a function of temperature. Equilibrium Monte Carlo simulations were performed on (110) slab, seven layers thick (with two surfaces), and periodic along the [110] and [001] directions. The geometry chosen for the current calculations represented an $(8 \times 8)$ surface unit cell. A mesh of ideal sites was laid out over each surface. The initial configurations were used an ideal missing-row structure, a

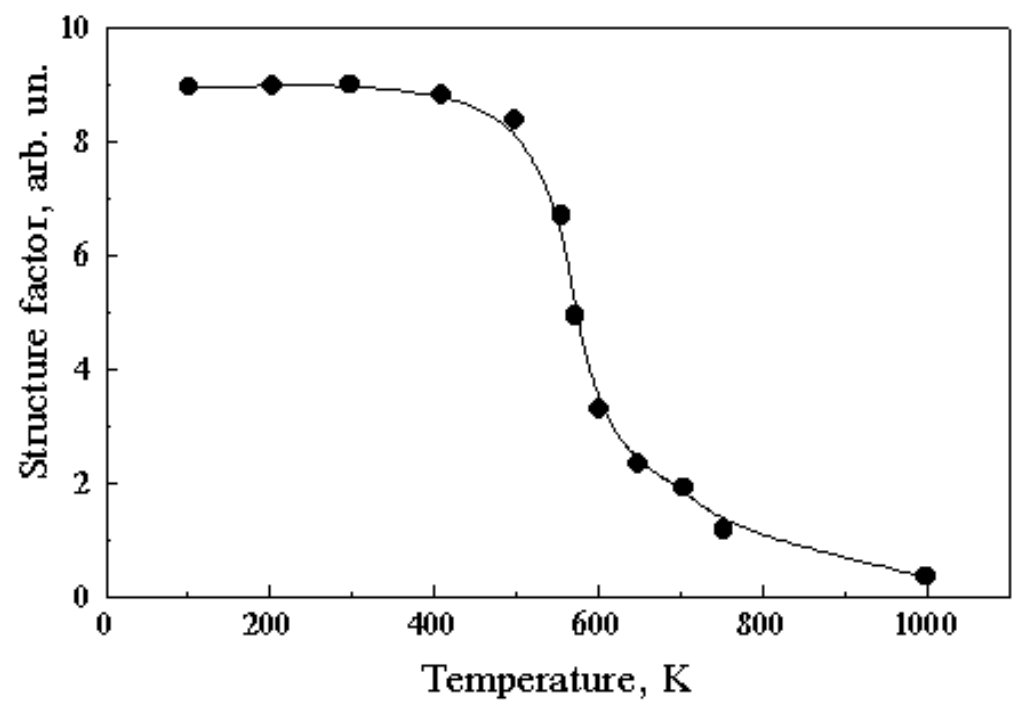

Figure 15. The structure factor for the $(1 \times 2)$ diffraction spot as a function of temperature on $\mathrm{Au}(110)$ [33]. 


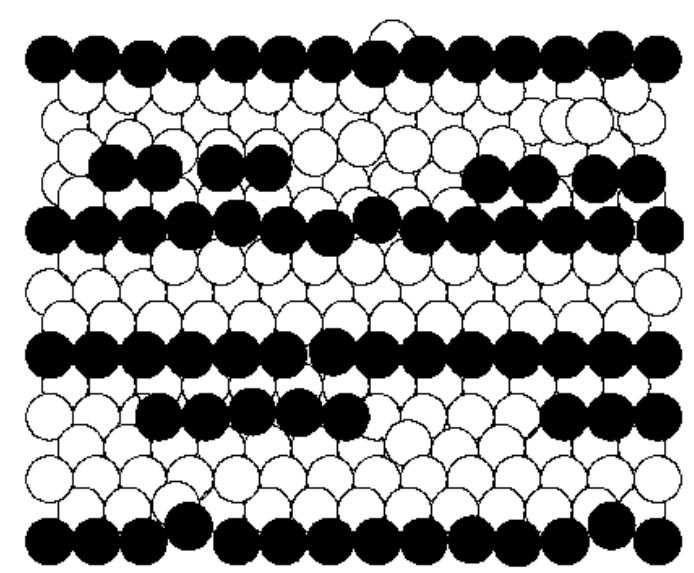

Figure 16. Snapshot of a randomly chosen structure after Monte Carlo simulation of the $\mathrm{Au}(110)$ surface [33].

$(1 \times 1)$ structure, and a random structure. In order to quantity the ordering the two-dimensional structure factor was computed. The structure factor was defined by

$$
S(\mathbf{k})=\left\langle\left|\sum_{j} \exp \left(i \mathbf{k} \cdot \mathbf{R}_{j}\right)\right|^{2}\right\rangle / N .
$$

The sum is over adatoms, $\mathbf{k}$ is the wave vector in reciprocal space, $\mathbf{R}_{j}$ is the position of $j$-th atoms, $N$ is the number of adatoms, and the angular brackets denote an average over configurations. The resulting structure factor for Au evaluated at the $(1 \times 2)$ diffraction condition as a function of temperature is plotted in Fig. 15. The transition temperature (defined as the inflection point in the curve) occurs at around $570 \mathrm{~K}$. The present calculations represent the first quantitatively realistic prediction of the orderdisorder transformation of a surface reconstruction. The disorder to the surface was characterized in this calculations by the second moment of the structure factor (i.e. the spot width) in the vicinity of the wave vector for the $(1 \times 2)$ symmetry. The temperature dependence is very similar to that for the experimental LEED spot width, which is fairly constant below $T_{c}$ and then increases rapidly with increasing temperature [18, 19]. The current theory showed, additionally, that the spot is not circular above $T_{c}$, but instead that the disorder perpendicular to the rows is stronger than that parallel.

Daw and Foiles on the base of analysis the structure factor for $(1 \times 2)$ structure as a function of temperature concluded that below the critical temperature the rows for $(1 \times 2)$ structure are generally long and coordinated, with some defects present. Well above the $T_{c}$ short-range order persists in the form of short chains of atoms along [110] direction 


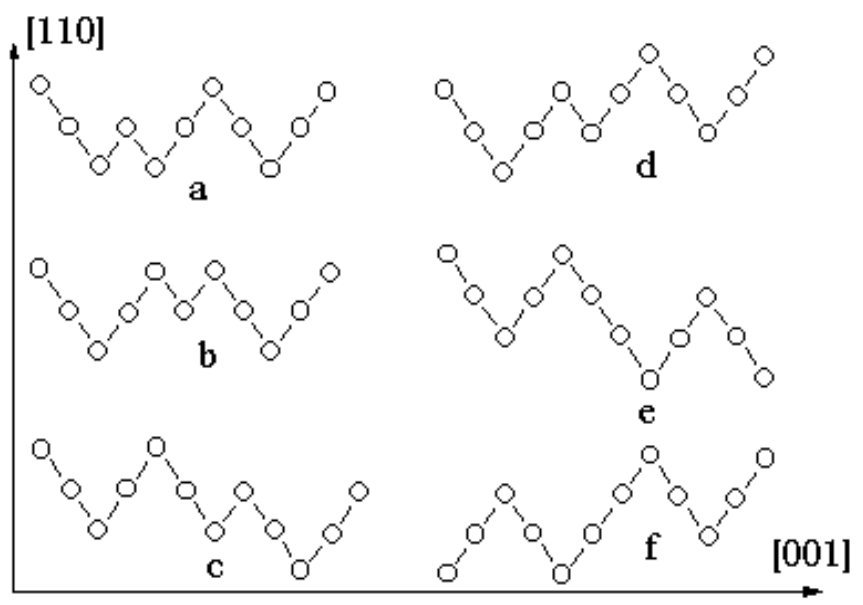

Figure 17. Excitations on the $(1 \times 2)(110)$ surface of $A u: a, b-I$ sing-like excitations; $\mathrm{c}-\mathrm{f}-$ roughening transition [34].

with little co-ordination among the rows. This was confirmed by snapshots of individual configurations generated by the Monte Carlo simulations, as exemplified in Fig. 16 [33]. The snapshot of Fig. 16 shows that the atoms in the EAM-Monte Carlo simulations do indeed move off of ideal lattice sites. EAM-Monte Carlo simulations illustrates the role of relaxation and vibrations since these effects are not included in the latticegas simulation within the framework of the Ising 2D-model, as examined in Ref. [18].

Bak [20] predicted an Ising model for deconstruction transition, however he did not consider another possibility, namely, the deconstruction might be a roughening transition. Indeed, Wolf et al. [17] observed roughness on their $\mathrm{Au}(110)$ samples and they have found $0.23<\beta<0.37$, arranger which is clearly above the Ising $2 \mathrm{D}$-exponent, e.g. $\beta=1 / 8$. However they found roughness at all temperatures and this can only be an effect of metastability.

Villain and Vilfan [34] first have presented the two-step disordering theory of the $\mathrm{Au}(110)-(1 \times 2)$ reconstructed surface, including a twodimensional Ising transition followed by surface roughening transition. From calculations of the free energy in terms of four energy parameters of meandering antiphase defects and surface steps (Fig. 17) Villain and Vilfan confirms the experimental observation that the deconstruction order-disorder transition of the (110) surface of Au and Pt belonging to the Ising universality class. Further, they predicted that at $T_{R}$, approximately $100 \mathrm{~K}$ above $T_{c}$, deconstructed surface should undergo a KosterlitzThoules roughening transition. The two-step disordering theory by Villain and Vilfan is consistent with most recently X-ray scattering study [27]. The more comprehensive data one can find in the recent review $[2,7,8]$ 
or in the appropriate references in the present paper [35-44].

The studies of the layer reconstruction in the clean metal surfaces has led to insights which motivated to investigate surface behaviour of single crystal metal alloys $[2,45,46]$.

\section{ACKNOWLEDGEMENTS}

This work was supported by INTAS-99 under Project \#01882.

\section{REFERENCES}

1. М. А. Васильев, Структура и динамика поверхности переходных металлов (Киев: Наукова думка: 1988).

2. M. A. Vasylyev, J. Phys. D: Appl. Phys., 30: No. 22: 3037 (1997).

3. F. Nieto, C. Uebing, and V. Pereyra, Surface Sci., 416: No. 1-2: 152 (1998).

4. N. Spiridis and J. Korecki, Appl. Surface Sci., 141: No. 3-4: 313 (1999).

5. D. Alfu and S. Gironcoli, Surface Sci., 437: No. 1-2: 18 (1999).

6. M. Lynch and P. Hu, Surface Sci., 458: No. 1-3: 1 (2000).

7. B. E. Nieuwenhuys, Surface Rev. and Lett., 3: No. 5-6: 1869 (1996).

8. B. E. Nieuwenhuys, Advances in Catalysis, 44: 259 (2000).

9. C. Becker, T. Pelster, M. Taneumura et al., Surface Sci., 427-428: No. 1-3: 403 (1999).

10. D. K. Captain and M. D. Amiridis, J. Catalysis, 184: 377 (1999).

11. M. A. Van Hove, R. J. Koester, P. S. Stair et al., Surface Sci., 103: No. 1: 189 (1981).

12. K. Heinz, E. Lang, K. Strayss et al., Appl. Surface Sci., 11-12: 611 (1982).

13. K. Heinz, E. Lang, K. Strayss et al., Surface Sci., 120: 401 (1982).

14. K. Heinz, G. Schmidi, L. Hammer et al., Phys. Rev. B, 32: No. 10: 6214 (1982).

15. D. Gibbs, B. M. Ocko, D. M. Zehner et al., Phys. Rev. B, 42: No. 12: 7330 (1990).

16. L. D. Roelofs, S. M. Foiles, M. S. Daw et al., Surface Sci., 234: 63 (1990).

17. D. Wolf and H. Jagondzinski, Surface Sci., 71: No. 1: 178 (1978).

18. J. C. Campuzano, M. S. Foster, G. Jennings et al., Phys. Rev. Lett., 54: No. 25: 2684 (1985).

19. J. C. Campuzano, G. Jennings, R. F. Willis et al., Surface Sci., 162: 484 (1985).

20. P. Bak, Solid State Communs, 32: 581 (1979).

21. D. E. Clark, W. P Unertl, and P. H. Kleban, Phys. Rev. B, 34: No. 6: 4379 (1986).

22. A. U. MacRae, Phys. Rev. B, 36: 2341 (1987).

23. H. Derks, J. Möller, and W. Heiland, Surface Sci., 188: 685 (1987).

24. T. T. Tsong and Q. Gao, Surface Sci., 182: 2257 (1987).

25. K. Dückers and H. P. Bonzel, Europhys. Lett., 7: No. 4: 371 (1988).

26. E. Riet van de, H. Derks, and W. Heiland, Surface Sci., 234: 53 (1990).

27. D. T. Keane, P. A. Bancel, J. L. Jordan-Sweet et al., Surface Sci., 250: 8 (1991).

28. J. Villain, Surface Sci., 199: 165 (1988).

29. J.-K. Zuo, Y.-L. He, and G.-C. Wang, J. Vac. Sci. Technol., 8: No. 3: 2474 (1990).

30. S. M. Foils and M. S. Daw, J. Vac. Sci. Technol. A, 3: 1565 (1985).

31. M. S. Daw and S. M. Foils, J. Vac. Sci. Technol. A, 4: 1412 (1986).

32. S. M. Foils, Surface Sci., 191: L779 (1987).

33. M. S. Daw, Surface Sci., 166: L161 (1986).

34. J. Villain and I. Vilfan, Surface Sci., 199: 165 (1988). 
35. T. Ali, A. V. Walker, B. Klötzer et al., Surface Sci., 414: No. 1-2: 304 (1998).

36. A. G. Makeev and B. E. Nieuwenhuys, Surface Sci., 418: No. 2: 432 (1998).

37. Q. Ge, D. A. King, N. Marzari et al., Surface Sci., 418: No. 3: 529 (1998).

38. M. A. Gonzbler, J. de la Figuera, O. R. de la Fuente et al., Surface Sci., 429: No. 1-3: L486 (1999).

39. V. Jahns, D. M. Zehner, G. M. Watson et al., Surface Sci., 430: No. 1-3: 55 (1999).

40. I. Vilfan, F. Lanzon, and E. Adam, Surface Sci., 440: No. 1-2: 279 (1999).

41. M. S. Zei and G. Ertl, Surface Sci., 442: No. 1: 19 (1999).

42. Meng-Sheng Liao, C. R. Cabrera, and Y. Ichikawa, Surface Sci., 445: No. 2-3: 267 (2000).

43. D. Passerone, F. Ercolessi, and E. Tosatti, Surface Sci., 454-456: No. 1-3: 634: (2000).

44. M. Salmeron and G. A. Somorjai, Surface Sci., 91: No. 2, 3: 373 (1980).

45. U. Bardi, Prog. Phys., 57: 939 (1994).

46. M. A. Vasylyev, A. G. Blashchuk, O. P. Hryshchenko, and N. S. Mashovets, Phys. Low-Dim. Struct., 11, 12: 115 (1999). 